Making clear what is fuzzy about community membership: A prototypic approach

Jordan Reed¹ (jreed28@depaul.edu), Ariel Jordan-Zamora ${ }^{1}$ (aajordanzamo@gmail.com), Crystal N. Steltenpohl² (cnsteltenp@usi.edu), \& Christopher B. Keys ${ }^{1}$ (ckeys@depaul.edu)

\author{
${ }^{1}$ DePaul University \\ ${ }^{2}$ University of Southern Indiana
}

Jordan Reed's ORCID ID: https://orcid.org/0000-0003-1212-082X

Acknowledgements: The authors would like to acknowledge Joshua Moore, Mark Belcher, and Robert Hubbs for giving feedback on the survey and for sharing the survey on social media. We also thank DieHard GameFAN, Evansville Esports, Game Informer, and others for publicizing the survey. Thank you to the Online Technologies Lab for continued help with the project. Lastly, we thank various FGC members on Reddit and elsewhere on social media for providing ongoing feedback on our data analyses and interpretation of results.

Data Availability Statement: The data that support the findings of this study are available in an Open Science Framework folder here https://osf.io/7ayz4/

Funding Statement: This project has no funders to disclose.

Conflict of Interest Statement: None of the authors have any commercial associations or financial interests that might create a conflict of interest in connection with this manuscript.

Ethics Approval Statement: This project was approved by DePaul University's Institutional Review Board. All participants provided written informed consent. 


\begin{abstract}
Aims: The critical task of positively identifying membership of the communities with which we work prior to initiating programs of research purporting to represent said communities is often forgone and treated as self-evident. This study demonstrates undertaking this task at the outset of a program of research by gathering member self-definitions of a relational, online and face-toface community- the Fighting Game Community.
\end{abstract}

Methods: Borrowing from social identity theory in social psychology, this study uses a prototypic approach and thematic content analysis with 319 open-ended descriptions of "good players" and "bad player" deviants.

Results: In general, having a growth mindset and winning games were the most crucial amongst divergent themes. Of equal interest, definitions were not consensual across the community; some directly conflicted with one another. These definitions represented a community with fuzzy boundaries, that exists more as a multi-layered tapestry than a black-and-white unit with sharply delineated boundaries.

Keywords: online, virtual, social identity theory, community membership, community definition 


\section{Making clear what is fuzzy about community membership: A prototypic approach}

Although membership is an important component of sense of community (McMillan \& Chavis, 1986), it is not well understood. For example, membership boundaries are less selfevident in relationally-defined communities than in geographically-defined communities (Gusfield, 1975; Hill, 1996; McMillan \& Chavis, 1986). While some relationally-defined communities may have centralized gatekeeping, in many, affiliation is less formal, which may result in community researchers using self-identification heuristics to determine membership. In these cases, defining membership boundaries is a fuzzy prospect, as no consensus on such boundaries exists (Jones, 2004). Community psychology methods often conflate self-selection into research participation with self-identification as a community member, which overlooks community members with meaningful reasons not to participate in research. Rarely do community researchers ask respondents to define the boundaries of a community of which they consider themselves members. We suggest researchers approach communities prepared to uncover a multi-layered tapestry of definitions and use methods of community membership definition sensitive to this possibility.

One way to counteract challenges in defining fuzzy or non-consensual boundaries is to assess membership definition without depending on concretely articulated boundaries or behaviors. Anchoring participant responses around community prototypes is an approach from social identity theory. A prototypical approach may provide a pathway toward defining membership without presupposing concrete community membership boundaries, while aligning with the way we perceive in- and out-group identity (Hogg \& Terry, 2000, Rosch, 1999). To explore the advisability of this approach in practice, we asked members of a (diverse in some 
ways, homogeneous in others) hybrid (involving online and face-to-face interactions) fighting game community to describe prototypical "good players" or deviant "bad players."

\section{An Avenue for Member-Defined Membership: Prototypicality}

Kloos and Johnson (2017) argue for the value of seeking potential synergic connections between community psychology and theories posited by other psychological disciplines, including social psychology. McMillan and Chavis (1986) used social psychological research as a basis for their conception of boundaries within community membership. They showcased research which shared intellectual roots with the now prevailing social identity theory in social psychology (Park, 1924).

In the spirit of Kloos and Johnson's suggestion, we examine our data through the lens of social identity theory. Social identity theory views group cohesion as a product of the “depersonalized, prototype-based interindividual attitudes” (Hogg \& Terry, 2000, p. 126) based on being members of the same group. This type of cohesion is based on social attraction, rather than personal attraction, which normally guides interpersonal relationships. This theory also predicts that, "where the prototype is consensual, certain people are consensually liked" (Hogg \& Terry, 2000, p. 126) and when prototypes are not consensual, individuals are less consensually liked. In some cases, an individual member of a community may believe that more other members agree with their sense of a prototype, or that more people align with one prototype than actually do, which is called the "false consensus effect" (Jones, 2004). Prototypes are less defined by definite boundaries, and more like "fuzzy sets," usefully understood by looking at members' varying notions of their category’s prototype (Oakes, Haslam, \& Turner, 1998, p. 75).

Prototypes are "abstracted representations of the central tendency, average or typical values of the members of a category" (Gilbert, Fiske, \& Lindzey, 1998, p. 407). While some 
prototypes are fuzzier or sharper than others, ordinarily there is no definite point at which a social boundary ends (Rosch, 1999). Most observers agree about the status of most objects or persons sharing common features of a category. However, if there are objects or persons at the margins of a category that may create confusion or disagreement, a prototypic approach adds value (Rosch, 1999). Prototypes can serve as representative exemplars (Rosch, 1999) around which other entities are associated based on "family resemblance" (Oakes, Haslam, \& Turner, 1998, p. 75) and are useful for defining the nature of group or community boundaries. Additionally, McMillan and Chavis (1986) suggested groups "use deviants to establish boundaries" (p. 9). Groups need deviants--bad members and scapegoats--to define themselves and solidify membership boundaries. Social identity theory suggests in-group deviants play a serious role in a group's understanding of its norms and prototype (Marques, Yzerbyt, \& Leyens, 1988).

Deviants can be pro-norm (extreme positive versions of the prototype; e.g., Captain America to the American soldier) or anti-norm (extreme negative versions of the prototype; e.g., a Nazi-sympathizing conscript to the same) (Abrams, Marques, Bown, \& Dougill, 2002). While pro-norm deviants are valued, anti-norm in-group deviants can be despised more than out-group members exhibiting the same behavior (Marques, Yzerbyt, \& Leyens, 1988; Rullo, Presaghi, \& Livi, 2015). This is called the "Black Sheep Effect" because such deviants, or "black sheep," pose a greater threat to group norms than non-members with the same characteristics. Targeting of marginal members as deviants can accentuate the prototypicality of others by contrast (Hogg \& Reid, 2006), and it is an oft-seen in-group prototyping behavior (Marques, Yzerbyt, \& Leyens, 1988). Relatedly, Fiedler (1964) suggested how a community identifies--and treats--in-group deviants serves as a useful indicator when defining community boundaries. Who they are 
contrasts with whom community members consider themselves to be, even more so than outgroup members (Rullo, Presaghi, \& Livi, 2015), and whether they are denigrated or tolerated provides insight to community priorities.

\section{Rationale}

We agree with McMillan and Chavis (1986) that "the most troublesome feature of [membership] definition is boundaries" (p. 9). Unfortunately, current sense of community literature has not accounted for the enhanced challenge of defining membership in informal, often relational, virtual and hybrid communities. Current methods do not address how deviant member conceptions influence experiences of membership, the ability for membership to exist on a gradient, or the often divergent, rather than consensual, manifestations of membership. Characterizing the qualities of a group member from that of a deviant or non-member using prototypes, by contrast, produces a picture that retains the gradient and disagreement between and around member categories. A prototypical approach welcomes diverse or conflicting conceptions of such categories to provide a more accurate and complex rendering of membership. To the degree it serves our understanding of the definition of community membership, prototypicality in the context of these communities is worth exploring. Further, comparing the prototypical member of the fighting game community (FGC) with in-group, antinorm deviants is a useful frame in this first attempt.

To this end, we explore the use of prototypes to understand membership definition for the FGC. Members of this community play fighting games, which involve face-to-face combat (akin to boxing or martial arts) using an avatar selected from a roster, like the games of Mortal Kombat, Guilty Gear, and Super Smash Bros. Fighting games can be played in arcades, on consoles like the PlayStation 4 or Xbox One, or on computers. In addition to gaming with others 
online, players convene in-person at arcade venues, local game stores, and regional, national, and international tournaments. Historically seen as a niche within the larger gaming community, in recent years, the FGC has received increased attention in part because of the rise in esports as an internationally popular activity. The community itself is divided on whether to embrace the "esports" label and the trappings that come with it (Lou, 2017). The FGC is often thought to be looked down upon by other gamers, the media, and the general public (Steltenpohl, Reed, \& Keys, 2018).

To apply this prototypic approach to understanding membership, we asked FGC members to characterize community members and deviants with these questions, "What makes someone a good player in the fighting game community?" and, "On the other hand, what makes someone a bad player in the fighting game community?" Formulating the prompts as "good player" and "bad player" emphasized the in-group role of "player" in the FGC around which the FGC is assembled. FGC member definitions of a "good player" are expected to reflect their conceptions of a FGC member prototype (Platow \& van Knippenberg 2001). These positive prototypes are likely to contrast with members' definitions of a "bad player," or in-group, anti-norm deviants. These deviants, according to social identity theory, are expected to serve as counterpoints to sharpen member prototypes and help illustrate where divisive differences may occur. Our research questions are:

(1) What characteristics do FGC members use to describe prototypes of "good players"?

(2) What characteristics do FGC members use to describe in-group, anti-norm, deviant "bad players"?

\section{Methods}

\section{Analysis}


Responses to the two focal survey questions were coded separately using thematic content analysis, in which researchers identify codes and themes aligning with the two study questions (Saldaña, 2012). Themes were identified inductively, derived from the data rather than preconceived themes or theory (Braun \& Clarke, 2006). Researchers applied three iterative coding phases coding to determine the final themes and code structure (Srivastava \& Hopwood, 2009). The integrity of the data is supported by thoroughly articulated methods, a reflexive statement of researcher positionality (provided below), reflexive memos, and a number of strategies to address the tension between participant meaning and the interpretation of researchers (Williams \& Morrow, 2009).

Credibility, confirmability, and dependability of researcher interpretations were enhanced using strategies recommended by Lincoln and Guba (1986) and Williams and Morrow (2009). Researchers worked toward credibility using peer debriefing and engaging with the community of interest. Peer debriefing included repeated discussions of analyses and findings among researchers over several months. Engaging with the community of interest took several forms. We asked FGC members for feedback on question relevance and language when designing the survey. Researchers also conducted a direct observation of an annual fighting game tournament. Moreover, researchers were invited to a private Facebook group for a local FGC venue by venue officials, and have attended events therein to gain an understanding of the communities. Fifth, researchers have corresponded with tournament organizers, FGC members, and two FGC members who are also researchers. Finally, researchers checked credibility by submitting a conference poster about the FGC to several FGC-oriented websites for feedback. The FGC members' affirming responses regarding the researchers, survey, and findings indicate researchers had established at least reasonable credibility through these six efforts. 
Confirmability was addressed using an open coding process wherein coders first familiarized themselves with the data, then took each analytical step inductively from the data. Between and during the open, axial, and selective coding stages, coders returned to the data to find confirming and disconfirming cases for their codes (Williams \& Morrow, 2009). Confirmability was further addressed using several analysts (MacQueen, McLellan-Lemal, Bartholow, \& Milstein, 2008; Williams \& Morrow, 2009). A team of researchers scrutinized codes and excerpts, then collaboratively judged, further refined, and ultimately agreed upon the suitability of codes. The fourth author served as an external auditor (Williams \& Morrow, 2009).

Dependability was evaluated using inter-rater reliability during the open coding phase (Lincoln \& Guba, 1986). Inconsistencies between coders were discussed throughout the coding process to develop consensus. Reliability was calculated using Cohen's Kappa; for good player codes, $\kappa=0.93$, and for bad player codes, $\kappa=0.81$.

\section{Researcher Positionality}

As previously described by Steltenpohl and colleagues (2018), the authors have varying levels of engagement with different gaming communities. The first author plays console games and is active on gaming-related Reddit forums. The second author has been involved in online gaming communities, played since she first was able to use a computer and has also competitively placed in racing games. The third author has been playing video games since she was four years old, has been involved with gaming-related forums since 2003, and has written for a few gaming websites. The final author has had experience gaming with family members. While none of the authors identify as FGC members, the first and third authors attended an anime fighting game tournament and the third author has personal and professional contacts within the FGC. All authors believe communities should be allowed to speak for themselves, and 
have made concerted efforts not to overinterpret qualitative responses. Having one member of the author team highly involved with gaming facilitated entry and participant recruitment. These different degrees and kinds of engagement enabled the authors to view the FGC and the results from diverse perspectives that, taken together, yield valuable insights.

\section{Respondents}

We collected 496 responses to an online survey distributed throughout social media platforms FGC members frequent, including Reddit and Twitter. The GameStop-owned magazine Game Informer also published a press release about the survey (Futter, 2016). On these surveys, 319 respondents answered both questions analyzed in this study; their demographic information is listed below. Regarding gender identity, $92.8 \%$ of respondents identified as male, $4.7 \%$ as female, $0.6 \%$ as trans, and $0.3 \%$ as nonbinary, while $1.6 \%$ did not respond. The mean age of respondents was $24.44(\mathrm{SD}=5.12)$. Regarding racial/ethnic identity, $58.2 \%$ identified as white, $13.3 \%$ as multiracial, $11.5 \%$ as Latinx, $9.6 \%$ as Asian, $6.5 \%$ as African American, and 0.9\% as Middle Eastern. Respondents reported having played video games in general for an average of 18.80 years $(\mathrm{SD}=5.32)$ and fighting games for an average of 11.21 years $(\mathrm{SD}=7.39)$.

\section{Materials and Procedures}

This study examined responses from a larger online survey about the FGC, including quantitative questions regarding community participation and qualitative questions regarding perceptions of outsiders' perceptions and portrayals of the FGC, and what factors respondents like and dislike about the FGC. The full survey and codebooks for this and other related studies can be accessed on the Open Science Framework under the title "2016 Fighting Game Community Online Survey" (Steltenpohl \& Keys, 2017). This analysis focused on responses to the open-ended questions, "What makes someone a good player in the fighting game 
community?" and, "On the other hand, what makes someone a bad player in the fighting game community?"

The survey was distributed through online venues native to the FGC, namely FGCrelated subreddits on Reddit, Facebook groups, well-known FGC members on Twitter, and gaming-dedicated websites. Participants were excluded if they were under 18 years of age or if they indicated a non-fighting game as their top community.

\section{Results}

This study sought to understand the good player prototypes and the descriptions of ingroup, anti-norm deviant members, i.e. "bad players," held by FGC members and used to define membership in their community. Though asked to define both good and bad players consecutively, many respondents were inclined to frame each on two sides of the same concept.

\section{Good Player Dimensions}

For the FGC respondents, what defines a good player is not determined solely on skill, which might be expected for a community focused on competition. Community members provided a wide range of qualities they considered imperative to the definition of a "good player." These ranged from their ability to employ a growth mindset, to perform well in competitive settings, to exhibit interpersonal kindness, to engage in the community. Also included in these responses was the notion that performance should not be a criterion.

Growth Mindset. The most frequently mentioned good player prototype quality was a focus on the process of playing the game and emphasizing love of the game, learning, and improving, over winning. Good players exhibited strong work ethic and practiced frequently, as evidenced by the statement, "They have the mindset that allows them to practice a lot." Good players make an internal attribution when things go wrong. They analyze and learn from their 
mistakes and weaknesses dispassionately. Additionally, good players are humble and acknowledge their abilities realistically; in other words, a good player "accepts their skill level." Further, respondents describe good players as those who solicit feedback, positive or negative, as another source of information to help improve their performance. They have a "readiness to learn and be taught $[\ldots]$ and humble to ask for help."

Absolute Performance (Git Gud). Respondents identified four major facets of absolute performance for good players: winning, skill, experience, and in-game adaptability. Put simply, a good player has a favorable win-loss record. One participant went so far as to specify a minimum ranking of eighth place at Evo, the largest international tournament for the FGC, as the standard for a good player.

A second facet of player performance involved general skill, which includes proficiency in executing moves and having an arsenal of technical maneuvers. The latter included knowing move combos (i.e., higher level execution tactics such as the use of a special power or heavy attack); strings (attacks that employ several button triggers in a specific order); the wise use of spacing; comebacks for certain moves; and other technical capacities such as "understanding hitboxes, the amount of frames an attack is[,] and knowing when to go on the offense and defense." Good players demonstrate their breadth of skill by "having a thorough working knowledge of every character's move-set" and/or by thoroughly mastering all the dimensions of a single character. Third, participants considered experience an indicator of a good player. The accumulation of the sheer volume and variety of circumstances and interactions encountered by a player was a value in itself, or in one respondent's words, "extensive experience playing."

Finally, respondents identified preparedness and mid-gameplay adaptability as an important aspect of a good player with one participant citing "the ability to adapt to the other 
player faster than the opponent." Respondents also specifically emphasized a player's aptness at playing "the mind game," referring to a player's ability to assess and deftly use the intra- and interpersonal psychological aspects of the game.

Interpersonal Kindness. Many respondents emphasized being a good player is dependent on how they treat others. They described interpersonal kindness as having a generally prosocial, positive, non-critical or not overly serious disposition, and as sportspersonship. One respondent explained this dynamic of sportspersonship, "You can be great at the game and beat anyone you play against, but if [you're] a poor sport it'll be a poor experience for people watching." Respondents focused on how good players keep frustrations to themselves. This dimension was not about performing under pressure, but about what happens after matches.

Prosocial behavior is the central element of interpersonal kindness. For some, prosocial behavior extended to demonstrating social skills, as when a player "makes friends easily." Further, meritorious players were willing to interact and compete with players not their equal in performance or skill, as a measure of magnanimity and unpretentiousness. Beyond differences in skill, respondents stated good players are tolerant and accepting of differences in general, whether between play styles, personalities, or identities.

Finally, players with a positive, not critical, disposition ensure the FGC experience remains fun; prioritizing enjoyment even over winning and losing with the ability to "step back and see the game as a game, have fun with it."

Community Engagement. A final element of being a good player was community building and maintenance. Respondents mentioned helpfulness towards the community generally, often through mentoring new players, volunteering at tournaments, and "keeping as many people involved as are interested." Community engagement differs from being kind; it 
represents service for the benefit of the community at large, rather than interpersonal manners. Good players pass on information to other players primarily as an investment in the community. In addition to contributing directly to a community of mutual self-improvement and information sharing, good players sustain the community beyond solely enabling others to play the game. Some good players put significant effort into streaming games, developing compilation videos of exciting or informative moments for the community, reporting on community events, and other activities. These may range from "[creating] combo videos, [filming] EVO, CEO[, and] other tournaments, to music and art." For some, just being there to communicate and reflect about the game can make someone a good player, as one respondent explained the importance of "how much they engage other players about in-depth game discussion."

Finally, good players may be judged by their effect on the community's permeability and relationship with other communities. For some, being community-engaged means being welcoming to outsiders and newcomers. A few respondents stated good players are "active in removing toxic and harmful members of the community." Several stated good players consciously ensure they are good FGC representatives by being mindful and considerate towards fans, taking care of members through talking and mentoring, and/or encouraging a strong sense of unity. Beyond the FGC, a few mentioned good players welcome contact with other gaming groups. These players abstain from "bashing these other communities;" as one respondent recommended, the rule is to "be cool to other communities and they'll be cool to you."

Anti-Performance. Here is but one example of the fuzziness of FGC members' membership boundaries and definitions of player quality. While a number of respondents mentioned winning and skill as characteristics of a good player, others specifically claimed these 
were not important to their definitions. One respondent explained, "Most of my favorite players aren't even those who you typically see finishing first or even in the top 8 of an event." Another echoed such tournament success was unimportant by saying, "Placements don't matter much to me." Some respondents considered winning completely irrelevant: "It's never about winning."

Similar degrees of unimportance were assigned to player skill as well; one respondent stated a player can be good for other reasons, "even if their skill level is low." While some considered skill irrelevant, they elaborated by saying: "I don't think that skill takes part of how good a player can be" and one stated simply: "not talent."

In sum, good players evidenced characteristics meant to showcase the FGC in a positive light and bring the community together. Respondents stated qualities of a good player included absolute performance, anti-performance, growth mindset, interpersonal kindness, and community engagement. Qualities ranged from being able to compete and place well in tournaments to supporting other players and helping the community. Some responses focused on one characteristic, others required a constellation of characteristics, and still others suggested the existence of more than one kind of prototypical good member within the FGC.

\section{Bad Player Dimensions}

Commonly but not uniformly, depictions of bad players included behaviors that were opposite to good player descriptions. Curiously, participants were more likely to give real-world examples of bad player behaviors than of good player behaviors. Even highly visible FGC players were described as expressing these negative qualities (see Figure 1). These FGC members included high-profile streamers (i.e., individuals who play and broadcast their actions live on the internet) and highly-ranked players. Further illustrating the diversity in definitions, a small subset of respondents refused to describe bad players, and some claimed there were no bad 
players in the FGC. Descriptions of bad player characteristics were grouped into five themes: being a scrub, trash, inconsiderate, elitist/isolationist, and "there are no bad players." For the first three themes, a deviant in the FGC could exhibit one or more characteristics at a time. These themes are presented in order of the frequency with which they were mentioned.

Scrub. The most common term used in defining a bad player was a "scrub," a pejorative term popular in gaming communities referring to a constellation of gameplay performance characteristics. Study participants noted scrubs do not try to improve themselves and instead are more likely to shift blame for their poor performance. Scrubs may be egotistical and quick to anger when losing. Scrubs are likely to take shortcuts or use cheap tactics, such as button mashing or using overpowered characters to win a match. These practices emphasize a win-atall-costs mentality and a lack of desire for growth, often accompanied by self-aggrandizing and ego-defensive communication about gaming actions.

Trash. $\quad$ Respondents described players who lose a lot or lack skill as "trash," which requires the player to (1) maintain a consistently poor loss-to-win ratio and (2) be generally unskilled. This is orthogonal to the attitudinal characteristic of being a scrub, as being trash is related purely to current skill. This label, despite its colloquial severity, carries much less negative connotation compared to "scrub" in the FGC. Scrubs can perform poorly or well, but the skills they develop are incidental to the pursuit of glory, and thus they may perfect the easiest skill set that produces victory. A trash player, on the other hand, does not understand the fundamentals of the game and often loses, but not because of one disposition or another. Thus, respondents consider the "trash" moniker as an indicator of a bad player that often applies only temporarily to any one individual, whereas the term "scrub" tends to imply something more permanent or dispositional. 
Inconsiderate. Inconsiderate players are commonly described as being antisocial and unable to play well with others. Bad players engage in excessive trash talking, which fosters a hostile environment. Inconsiderate players lack self-awareness and alienate others by being mean, disrespectful, or generally hurtful. One FGC member stated these players engage in "non stop complaining, starting drama, racism, sexism," and tend to be bitter and try "to get other people involved in their own crap." Inconsiderate players focus on themselves and, intentionally or not, hurt others to divide the community.

Respondents described inconsiderate players as "cancer," a term used for players who make the community a hostile environment. Negative attitudes were a common marker for inconsiderate players as they cared more about, as one respondent said, creating "beef," drama, and confrontations, than playing with the community. In contrast with the scrub theme, being inconsiderate was related to the attitude players bring to interpersonal relationships, rather than to gameplay performance.

Elitist/Isolationist. The final defining characteristic of a bad player is elitism and/or isolationism. Elitists are unhelpful, dismiss games they do not play and attack their communities, shun new players, and belittle other players at every opportunity.

A common complaint regarding elist and isolationist players was selfishness. Selfish players "[contribute] nothing" and "sponge off of others." Elitist players were described as "hat[ing] on the other fighting games people play that are not their favorite fighting games." For example, elitist players treat members of "Smash Brothers [like] they aren't part of the community." Elitist players may do this to their own community as well by "[threatening] to leave if the [developers] don't do X,Y,Z," "mak[ing] fun of others, mak[ing] it personal, bully[ing]" and/or "say[ing] bad things about the game instead of promoting [it] in a positive 
light." Elitist and isolationist players also negatively impact their own communities by keeping new players out of the game by making them feel like a burden and inferior to the community.

No Bad Players. While many respondents described qualities indicative of a bad player, there was also a subset of individuals that refused to label anyone in the community as bad. These respondents insisted bad players did not exist; everyone started off at the bottom before climbing their way up. Respondents did not specify if this was referring to skill or to other possible measures of a good player. These respondents believe it takes all kinds and the FGC should not be exclusive. They reinforced the idea every player once had negative qualities but can improve by learning how to play and through positive interactions with the community.

\section{Good and Bad Player Correspondence}

Themes respondents used to describe a good player typically had a corresponding and opposite theme to describe a bad player (see Figure 1). Responses composing the absolute performance theme were rough counterparts to those making up the trash theme. Losing is the clear corollary to winning, while "no fundamentals" and "cheap tactics" are the opposite of “skill.” Similarly, a "scrub” uses ego-preserving alternatives rather than a growth mindset. Specifically a scrub's "short fuse" represents the absence of an ability to "control arousal." Also, shifting blame is the opposite of analyzing one's flaws and lost games, accepting one's skill level, and seeking advice. Subthemes for "interpersonal kindness" and "inconsiderate" had the clearest 1:1 connections. Being prosocial vs. antisocial, exhibiting sportsmanship vs. a lack of sportsmanship, and being positive vs. being negative/serious/critical reflect determinants of prototypicality that appear to be especially binary to respondents. Binaries also exist between a proportion of the sub-themes for the elitist/isolationist and community engagement themes; 
specifically helpful vs. unhelpful, cool vs. harsh to other communities, and being a good community representative vs. representing the community poorly.

However, there were a number of good-player sub-themes that did not have a counterpart. Respondents mentioned experience as a meritorious facet of absolute performance on its own, where there is no mention of this in bad player responses. Similarly, there was no corollary to conversing about the game mentioned in the deviant descriptions. To embody community engagement, respondents mentioned that good players should be willing to converse about the game, and possibly create content for other players, which were two further sub-themes without an opposite. One explanation for themes without an opposite would be that they refer to behaviors that some respondents consider value-adding but not disqualifying from being a good member when the behavior is absent. Inexperience not being a defining factor of a bad player is supported by respondents' focus on players' ability to learn and to accept their skill level in the "No Bad Players" and "Growth Mindset" themes, and "Elitism/Isolationism" in the bad player themes. Some respondents may also view subthemes of conversing about games and creating content as tertiary to their conceptions of membership.

\section{Discussion}

Responses helped us begin to better understand how FGC members define FGC membership prototypes and deviants. There was a high degree of inverse correspondence between good and ingroup deviant player themes. Yet definitions of both varied in meaningful ways that are useful for gaining a glimpse into what is pertinent when respondents make distinctions between good player and deviant. Respondents characterized "good players" as those who exhibit acceptable performance, a growth mindset, interpersonal kindness, and community integration. "Bad player" in-group deviants acted like "scrubs," and were "trash," inconsiderate, 
and elitist/isolationist. Additionally, in the good player descriptions some respondents specifically stated that winning was not an important characteristic of a good player (antiperformance), and in the bad player descriptions some respondents stated, "there are no bad players." After reviewing how respondents differ in the number and relative importance of characteristics they endorse for either element (good player and deviant) of membership definition, we conclude the prototypic approach added value. This approach is helpful when characterizing informal, relational hybrid community boundaries without erroneously simplifying the varied patchwork of their composition and values.

\section{Salience of Deviants}

This study considered the question of how FGC members define membership using prototypes of good players and descriptions of in-group, anti-norm, deviant, bad players. Of particular interest is the one occasion ("scrub" versus growth-mindset) where a description of a bad player is more consensual and crystallized than that of the corresponding description of a good player, supporting the notion that defining deviants is important for membership definition

(Hogg \& Reid, 2006; McMillan \& Chavis, 1986; Rullo, Presaghi, \& Livi, 2015). As proposed by Marques and colleagues (1988), denigrating in-group deviance serves as a referent to differentiate and protect the group's most often positive prototype. In the case of the processfocus theme, a chorus of respondents described a loathed deviant in the oft-seen and poorlyregarded player archetype: the scrub. The denigration of the scrub contrasts with the understanding afforded to a temporarily unskilled or frequently-losing member who does embody a growth mindset. In the case of the scrub, we see a member typified by adherence to one standard of a good player (trying to achieve absolute performance), while failing another (refusing to employ a growth mindset or be self-critical). In the data, there is no similar 
pejorative term associated with a player trying faithfully to improve (achieving growth mindset) but still lacking (failing absolute performance). This absence suggests that to some respondents, being a good player in terms of having a growth mindset is more essential than being a good player in terms of skill.

Parallels can be found in Fiedler's contingency theory, which posits the priorities of an individual can be displayed in how they envision their least-preferred coworker (Fiedler, 1964). If a person rates their least-preferred coworker high on friendliness, relationships are more important to the rater than performance. Conversely, if they rate their least-preferred coworker lower on efficiency, they prioritize accomplishing tasks. In this study, there was a minority of members who suggest winning or being skillful is not important, combined with a similar minority who would hesitate to call anyone a bad player. This group of members likely favor what Fiedler would call a relationship orientation.

\section{Divergent Expectations}

The abstractness and variance seen in both good membership prototype and in-group anti-norm deviant descriptions with these respondents challenges the applicability of clear insider/outsider boundaries for all communities, and instead serves to exhibit a case in which familial gradation (Oakes, Haslam, \& Turner, 1998) and degree of consensus (Hogg \& Terry, 2000) influence member definition. In this study, only two concrete definitions for a good player were offered (tournament placings and win-loss ratios) that could permit a researcher to apply a community member's standard with confidence from the outside. However, the consensus of respondents did not support these two standards. The same could be said for descriptions of a "scrub" player; while conceptions of said players were more crystalized than those for other 
themes, there was still divergence and a large proportion of respondents did not mention the concept of "scrubs" at all.

The remaining fuzzier means by which FGC members describe themselves do not permit such unquestioned delineation, whether attempted by a researcher or by the members using their own definitions. These fuzzier descriptions well-typify the depersonalized and abstracted format that social identity theory states are used in understanding group membership (Oakes, Haslam, \& Turner, 1998). For example, judgement about whether any player demonstrates a growth mindset, interpersonal kindness, community engagement, or even sufficient skill is a matter of degrees.

Interestingly, beyond the two participants who suggested specific tournament placings, no respondents offered a cutoff for a characteristic that could be used to indicate a good player or a deviant. This general lack of clarity supports the prototypic approach, which suggests the existence of a defining line or perfect example is a misconstrual of the grey area we perceive when forming group identities (Rosch, 1999). Rather, social identity theory claims we judge the membership of an individual example on a familial gradient (Rosch \& Mervis, 1975) based on subjective appraisal of that individual in comparison to individuals on prototypical characteristics. Such a comparison permits us to judge whether someone is more or less a member; there is usually no agreed upon point where membership begins and ends (Oakes, Haslam, \& Turner, 1998; Rosch, 1999).

Meaningful trends in membership definition with relational hybrid communities can be gleaned from considering respondents' perspectives about the number of characteristics their prototype includes and their relative importance. Respondents described being a good player as defined by one characteristic or by a combination of important behaviors with similar frequency. 
In some cases of the latter, listed behaviors were complementary (i.e., that the player be skilled and hardworking). In other cases, a respondent's vision of a good player required less directly connected behaviors in tandem (e.g., that they were skilled and kind). Finally, a smaller subset of respondents explained good players can fit one of many roles, and one need not exhibit all "good" behaviors to qualify as a good player. This final set of respondents held the perspective FGC members should and do fill multiple roles, and that specializing in various roles is more realistic than using one dimension to judge membership in the FGC. This complexity of good player categories supports the notion that relational communities are less likely to share a consensus on membership definition (Gusfield, 1975; Jones, 2004).

\section{False Consensus}

That respondents present diverse descriptions of a good and bad player leads us to question whether there is a consistent conception to extract from the respondents' accounts that could offer a high degree of certainty distinguishing whether any given player is a good or bad player in the FGC. It could be more likely that members of the FGC, due to unique contexts and being a relational hybrid community in general, are especially susceptible to the false-consensus effect. The false consensus effect is when a group member believes that more other members agree with their sense of a prototype, or that more people align with a single prototype when the reality is much more varied and nuanced (Jones, 2004). Respondents variably claimed prototypicality was conditional on one factor, on multiple necessary but individually insufficient factors, on a "one-of-the above" appraisal, and/or on whom you ask. That these respondents indicated a differing number of conditions and disagreement on whether it was an and or or proposition might be more representative of how membership is actually perceived in the many communities we study, however. 
A crucial consideration for this study's design is that each respondent self-reported as an FGC member, as is the case with the FGC in general. There is no review body with authority to grant membership status. Thus, there are likely fewer systematic, exhaustive, reflexive processes that allow the community to census or monitor the range of behaviors and characteristics exhibited by its members. This lack of reflexivity may lead to false consensus.

Additionally, "good player" and "bad player" behaviors were based on members' experience in the community, rather than on some unified guiding document from the top-down. Further, we did not assess definitions resulting from a common forum for collective normbuilding. We propose that for these reasons, only the broadest definition of good or bad players is inclusive of the definitions offered by all respondents. While there are certainly trends, there is no definitively right or wrong answer; there is no one prototype of a good or bad player in the FGC. Yet the members consider themselves a community. We have no doubt other communities studied in community psychology are similarly fuzzy in membership definition. One may argue geographic communities or exhaustive modeled networks have more definite member prototypes and thus applicable standards for boundaries. Nonetheless, relational communities do not abide by the same rationales by which geographic community boundaries are often delimited. In the many cases where membership is not explicitly formalized, fuzziness may abound.

\section{Emergent inadequacy of player-focus}

This study endeavored to first invite the FGC to define itself to dictate the strategies and scope of the following program of research. In so doing, the research team has demonstrated the danger of forgoing this foundational effort. The research team presumed a breadth of member characteristics and designed the membership questions to elicit them, yet still the prompt itself illustrated the limits of our initial conceptualization. 
Even in response to a question about "players," participants told us that one need not even play fighting games to be a member of the fighting game community. Others did not make such a clear contrast, but still emphasized a wide landscape of roles that could be equally or more important to playing in their definitions.

Based on the respondents who did the work of reframing the question, we anticipate that other respondents could have provided different definitions, were they all asked to define "what makes someone a good member in the fighting game community?" rather than, "what makes someone a good player in the fighting game community?" Without said respondents reacting to the language of our prompt, we would never have been informed that the definitions could differ, and of the whole landscape of the FGC. Without asking the question first, as is the case with many programs of research, our work would have never benefited from this reaction.

\section{Limitations}

Due to its exploratory nature, the survey contained many other questions aside from the topic of interest, some of which came before the membership questions and which may have influenced attrition rates over the survey questions. While providing a broad map to capitalize on, open-ended qualitative survey questions in general, and those studied here in particular, should be regarded as more inclined to gather a breadth rather than a depth of information and limited by no facility for clarification. Some respondents also gave brief answers, which made it difficult to capture nuances in their prototypes and definitions. That the survey was written only in English may have also skewed the perspectives represented therein.

\section{Future Research}

It is clear that future research should ask respondents "what makes a good member", "what makes a bad member" and "what makes someone a nonmember" of the FGC. But beyond 
that, the variance in participant definitions potentially belies distinctions between undistinguished factions in the FGC. Although spaces inhabited by the community commonly speak in terms of the FGC at large, perhaps members who play different games, who focus on one game or several games, or who focus on different objectives define the FGC differently. Future research should explore deeper into respondent behaviors and their situation within the larger group, to interpret their definitions of the larger group with that positional data in mind.

With the benefit from the broadly captured yet brief responses from the open-ended survey questions studied here, semi-guided interviews with respondents who are selected using newfound indications of community homogeneity would permit more meta-reflection on membership definition. Of particular interest is how respondents in different positions regard the membership definitions offered in this exploratory study.

Qualitative exploration of connection between judgments of prototypicality and selfreported psychological sense of community would be especially elucidating. Whether a person who perceives themselves as more prototypical is likely to report a higher sense of community could help explain group cohesion and schisms.

\section{Conclusion}

This study considered the nature of community membership. Using an approach borrowed from social psychology, we have employed questions which frame fighting game community membership based in terms of prototypicality and in-group deviance. This approach articulates the ideal player and its contrast, and judges membership based on degrees of congruence with the ideal. While many respondents provided definitions of membership in general, some were undoubtedly providing definitions of playership only, which may or may not represent their view of FGC membership as a whole. 
We learned some players venerate pure performance, while some prioritize prosocial and community-centric behavior. We investigated whether FGC members' self-reported conceptions of a prototypical good player and a deviant bad member were consensual, we learned some players require many characteristics to consider someone a "good player" while others only care about one characteristic. Still others believe there are many roles and competencies demanded by the community, each of which need to be filled.

Using a prototypicality and deviance approach permitted us to preserve some intricacies of respondents' varying perspectives amidst fuzzy and diverse conceptions of community membership. This study demonstrates one possible alternative to rigid boundaries or to solely self-identification as bases for membership. Rather, it is possible community members prioritize different prototypes, attend to different characteristics , and/or interpret characteristics differently from one another. Especially in cases where there is no centralized gatekeeping role, this diversity of perception and evaluation, rather than necessarily translating feelings of membership into homogeneous group composition, diffuses a sense of membership so that the affiliations to whom it applies are varied.

This more complex rendering of community membership suggests that in much the same way that an individual's sense of community reflects their involvement in a "tapestry" (Brodsky, 2016) of communities, it is possible individual communities are a patchwork of a different sort, with membership composed of the overlapping and contrasting conceptions of each member. Learning more about what these patches are, how they interrelate, and whether exogenous variables impact this relationship, can help us to refine our understanding of FGC membership and community membership in general. The unanticipated emergent responses and participant revelations regarding the limitations of our initial framing of the question of membership 
demonstrate how thoroughly the practice of allowing a community to define itself first can impact the trajectory and thus validity of a program of research. 


\section{References}

Abrams, D., Marques, J. M., Bown, N. J., \& Dougill, M. (2002). Anti-norm and pro-norm deviance in the bank and on the campus: Two experiments on subjective group dynamics. Group Processes \& Intergroup Relations, 5(2), 163-182.

Braun, V., \& Clarke, V. (2006). Using thematic analysis in psychology. Qualitative Research in Psychology, 3(2), 77-101.

Brodsky, A. E. (2016). Bridging the dialectic: Diversity, psychological sense of community, and inclusion. American Journal of Community Psychology, 58(3-4), 284-293.

Fiedler, F. (1964). A contingency model of leadership effectiveness. Advances in Experimental Social Psychology, 1, 149-190.

Futter, M. (2016, January 19). DePaul University seeks fighting game community members for psychology research. Game Informer. https://www.gameinformer.com/b/news/archive/2016/01/19/depaul-university-seeksfighting-game-community-members-for-psychology-research.aspx

Gilbert, D. T., Fiske, S. T., \& Lindzey, G. (1998). The handbook of social psychology. Boston: McGraw-Hill.

Gusfield, J.R. (1975). Community: A critical response. New York, NY: Harper.

Hill, J.L. (1996). Psychological sense of community: Suggestions for future research. Journal of Community Psychology, 24, 431-438.

Hogg, M. A., \& Reid, S. A. (2006). Social identity, self-categorization, and the communication of group norms. Communication Theory, 16(1), 7-30.

Hogg, M. A., \& Terry, D. J. (2000). Social identity and self-categorization processes in organizational contexts. The Academy of Management Review, 25(1), 121-140. 
Lincoln, Y. S., \& Guba, E. G. (1986). But is it rigorous? Trustworthiness and authenticity in naturalistic evaluation. New Directions for Program Evaluation, 1986(30), 73-84.

Lou, G. (2017). Integrity vs. esports -- Do money and TV threaten the fighting game community's identity? EventHubs. https://www.eventhubs.com/news/2017/nov/05/integrity-vs-esportsdo-money-and-tvthreaten-fighting-game-communitys-identity

Macqueen, K. M., McLellan-Lemal, E., Bartholow, K., \& Milstein, B. (2008). Team-based codebook development: Structure, process, and agreement. Cultural Anthropology Methods, 10(2), 31-36.

Marques, J. M., Yzerbyt, V. Y., \& Leyens, J. (1988). The "black sheep effect": Extremity of judgments towards ingroup members as a function of group identification. European Journal of Social Psychology, 18(1), 1-16.

McMillan, D. W., \& Chavis, D. M. (1986). Sense of community: A definition and theory. Journal of Community Psychology. 14, 6-23.

Oakes, P., Haslam, S. A., \& Turner, J. C. (1998). The role of prototypicality in group influence and cohesion: Contextual variation in the graded structure of social categories. In S. Worchel, J. F. Morales, D. Páez, \& J.-C. Deschamps (Eds.), Social Identity: International Perspectives (pp. 75-92). Thousand Oaks, CA: Sage Publications, Inc.

Park, R. E. (1924). The concept of social distance as applied to the study of racial attitude and racial relations. Journal of Applied Sociology, 8, 339-344.

Platow, M. J., \& van Knippenberg, D. (2001). A social identity analysis of leadership endorsement: The effects of leader ingroup prototypicality and distributive intergroup fairness. Personality and Social Psychology Bulletin, 27(11), 1508-1519. 
Rosch, E. (1999). Principles of categorization. Concepts: Core Readings, 189.

Rosch, E., \& Mervis, C. B. (1975). Family resemblances: Studies in the internal structure of categories. Cognitive Psychology, 7(4), 573-605.

Rullo, M., Presaghi, F., \& Livi, S. (2015). Reactions to ingroup and outgroup deviants: An experimental group paradigm for black sheep effect. PLOS ONE, 10(5), e0125605. http://doi.org/10.1371/journal.pone.0125605

Saldaña, J. (2012). The Coding Manual for Qualitative Researchers. London, England: SAGE Publications Ltd.

Steltenpohl, C. N., \& Keys, C. B. (2017). Fighting Game Study. http://osf.io/qtmwn

Steltenpohl, C. N., Reed, J., \& Keys, C. B. (2018). Do others understand us? Fighting Game Community member perceptions of others' views of the FGC. Global Journal of Community Psychology Practice, 9, 1- 21.

Srivastava, P., \& Hopwood, N. (2009). A practical iterative framework for qualitative data analysis. International Journal of Qualitative Methods, 8, 76-84. http://dx.doi.org/10.1177/160940690900800107

Williams, E.N., \& Morrow, S. L. (2009). Achieving trustworthiness in qualitative research: A pan-paradigmatic perspective. Psychotherapy Research, 19(4-5), 56-582.

\section{Figure 1}


Good and Bad Player Correspondence

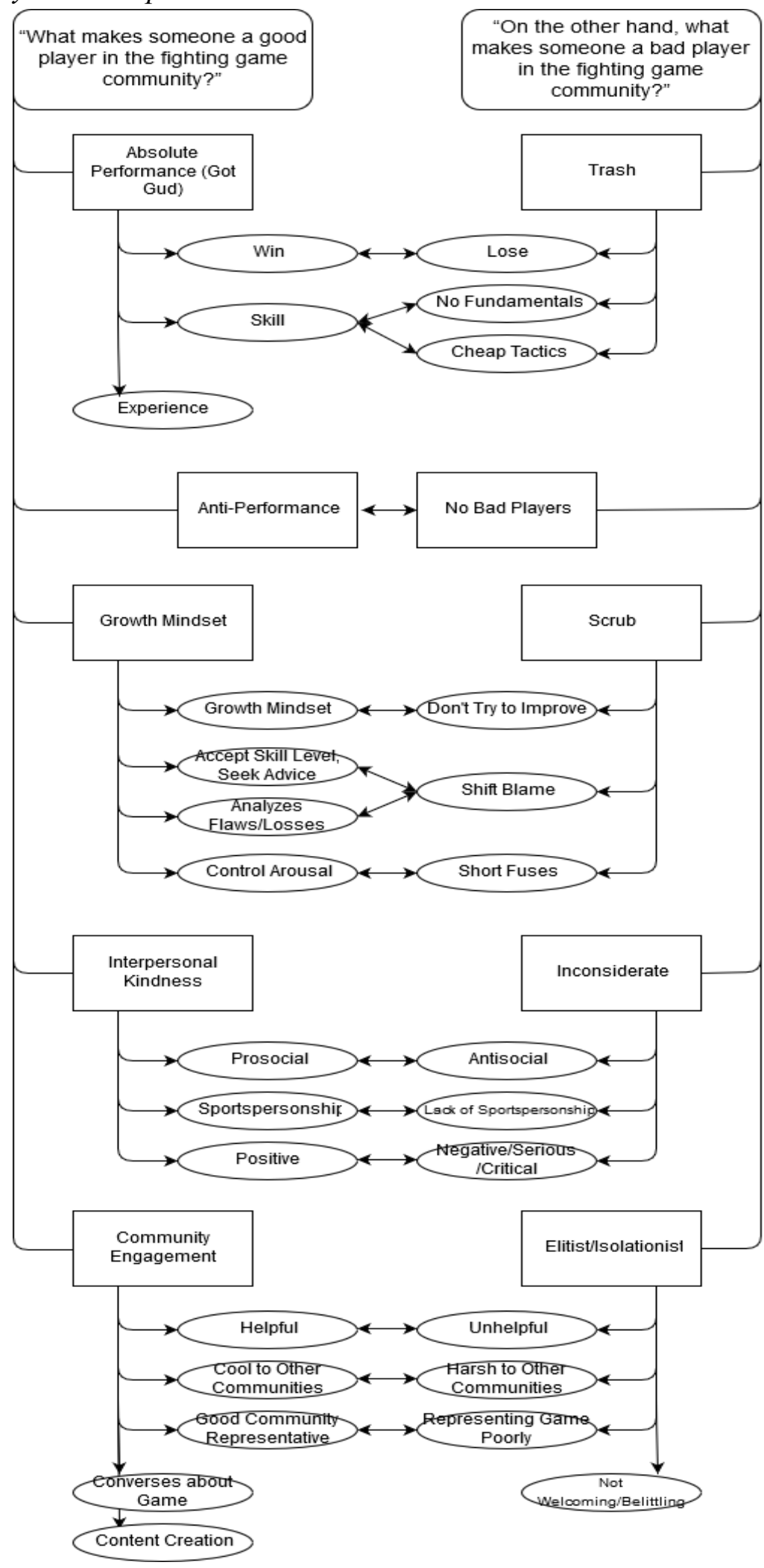

